\title{
EFFECT OF NONADIABATIC STATES CLOSE TO BRILLUOIN ZONE FACE ON VIBRATIONAL SPECTRUM
}

\author{
E. V. Zarochentsev \\ Donetsk Physico-Technical Institute, National Academy of Sciences of Ukraine, \\ 72, R. Luxemburg Str., Donetsk, UA-340114, Ukraine
}

(Received May 16, 1998)

\begin{abstract}
Nonadiabatic states of electron-ion system close to Bragg plane play an important role in forming vibrational spectrum singularities in a crystal. In ordered matter the presence of nonadiabatic states causes drastic transformation of the behavior of nondiametral singularities near electron topological transition. This effect is most pronounced when phonon momentum connects two reconstructed parts of electron spectrum near a Bragg plane. Some polarization operator singularities lead to nonanalytic behavior of a phonon group velocity as a function of momentum under the conditions. These conditions are obtained in the present paper.

Key words: electron-topological transition, nonadiabatic states, polarization operator, electronphonon interaction, vibrational spectra.

PACS number(s): 63.20.Dj; 63.20.Kr
\end{abstract}

\section{INTRODUCTION}

The nonadiabaticity in a crystal manifests in two ways: as interaction of electrons with ion vibrations and as lattice anharmonisms. The first effect attracts the permanent attention relatively to the problem of $H-T_{c}$ superconductivity (see review [1]). Then, a study of the nonadiabatic effects (in harmonic approximation) in crystals is of a great interest especially when Fermi surface is close to the Bragg plane (Brilluoin zone face). Another problem requiring the consideration of the Fermi surface to a Bragg plane proximity effects is the problem of the peculiarities in the properties of crystal under high pressure up to insulator-metal transition pressure.

One can observe the effects of nonadiabaticity in phonon spectra in the easiest way. The present paper is devoted to the study of phonon curves singularities caused by the proximity of the Fermi surface to the Bragg plane.

There is a narrow layer $\sim \hbar \omega_{D}$ of nonadiabatic states in the vicinity of the Fermi level in metals and narrow gap semiconductors. One usually neglects the contribution of this layer due to a small phase volume of it. These states should be taken into account with closing the fundamental band gap $E_{g}$ (e.g.,in strongly compressed insulators). Electron velocity in these states has the same order of magnitude as the phonon velocity has. The consideration of this case is important because the Fermi surface to the Bragg plane proximity is a more usual case than the exotic one. The development of general theory of nonadiabatic states is also necessary for the investigation of such phenomena as superconductivity, lattice instability etc.

The adiabatic approximation is the successful and well developed both theoretically and experimentally approaches to investigate vibrational spectra of insulators $\left(E_{g}>\hbar \omega_{D}\right)$ at the present time. The adiabatic approx- imation in lattice dynamics was also established to be valid for metals (see reviews $[2,3]$ ). The validity of adiabatic approximation for metals (also for narrow gap semiconductors $E_{g} \leq \hbar \omega_{D}$ ) is based on few nonadiabatic states in the layer with the energies $\sim \hbar \omega_{D}$ as compared to the number of all the electron states with the energies $\sim E_{F}$.

Approximately at the same time Kohn showed that in metals the $\left(q-2 k_{F}\right) \ln \left|q-2 k_{F}\right|$ singularity in screening at $q=2 k_{F}$ ( $q$ is a momentum transferred to an electron) gave rise of a similar singularity in vibration spectrum [4]. At first the Kohn anomaly (or something that one regards as Kohn anomaly) had been observed experimentally in the behavior of phonon dispersion curves of $\mathrm{Pb}[5]$.

Diametral singularities belonging to a systems with a spherical Fermi surface were discussed in $[4,6]$ neglecting electron phonon interaction. The singularities at other values of phonon momentum $\mathbf{q}$ may also occur along with diametral Kohn singularities at $|\mathbf{q}+\mathbf{g}|=2 k_{F}$ as the Fermi surface is reconstructed strongly near the Bragg plane ( $\mathrm{g}$ is reciprocal lattice vector). The hierarchy of many-particle singularities in electron multipolar diagrams had been established in [2] taking into account impaired indirect interaction of ions through conductivity electrons. The contribution of nondiametral Kohn anomaly was always small as compared to the contribution of the three-particle one [7].

Anomalies in the phonon spectrum are most seen clearly on the curves of group velocity $\partial \omega / \partial \mathbf{q}$ as a function of the momentum. Precious measurements of the $\partial \omega / \partial \mathbf{q}$ versus $\mathbf{q}$ of $\mathrm{Al}$ are given in [8]. Both the diametral Kohn anomalies and a number of the nondiametral ones which due to the electron spectrum reconstruction near the Bragg plane had been found there.

The significance of considering nonadiabatic states is already seen in the frames of near free-electron approximation [9, 10]. Qualitative changing in the phonon group 
velocity behavior over the results of adiabatic approximation appear in the vicinity of $q \sim 2 k_{F}$. A consistent consideration of nonadiabatic states leads to the renormalization of $\partial \omega / \partial \mathbf{q}$ at $q \sim 2 k_{F}$. This renormalization removes the logarithm divergences existing in adiabatic theory. The group velocity has pole singularities instead the logarithm ones (see below).

Anomalies in the phonon spectrum of niobium are something similar to the ones considered above [11-13]. The phonon spectrum of niobium contains a number of anomalies, including phonon group velocity anomalies as shown in [13]. The calculation for niobium [11] showed the electron structure to contain the domains of a finite size for which the adiabatic approximation was violated. The phonon spectrum singularities at $10 \mathrm{MeV}$ for $\mathbf{q}$ [001] and at 10-21 MeV for $\mathbf{q} \|$ [110] may be explained by nonadiabaticity of niobium electron-phonon system as follows from [11].

The division of electron and vibrational degrees of freedom of electron-ion system is impossible when the effect of nonadiabatic states on vibration spectra is investigated. So, we apply a self-consistent description of the crystal by the Green function technique.

\section{DYNAMICAL SUSCEPTIBILITY}

We show briefly the singularities in vibrational spectrum to be determined by the singularities of the polarization operator $\pi\left(\mathbf{q}, \mathbf{q}^{\prime} ; \omega\right)$. We use the results obtained in $[9,10]$. Let us consider an equation for the Green func- tion $\hat{\mathcal{D}}_{\mathbf{q}}(\omega)$ of ion displacements only. Its poles determine lattice vibration spectrum. We have for the representation of $\hat{\mathcal{D}}_{\mathbf{q}}(\omega)$ a standard form

$$
\hat{\mathcal{D}}_{\mathbf{q}}(\omega)=\left[\hat{I} \omega^{2} M-\hat{D}(\mathbf{q} ; \omega)\right]^{-1},
$$

where $\hat{\mathrm{I}}$ is the unit matrix, $M$ is the mass of the ion, and $\hat{D}(\mathbf{q} ; \omega)$ is a generalized dynamical matrix of a crystal depending on the unknown lattice vibration frequency $\omega$

$$
\hat{D}(\mathbf{q} ; \omega)=\hat{D}_{i}(\mathbf{q})+\hat{\Pi}(\mathbf{q} ; \omega) .
$$

In eq. (2) the $D_{i}^{\alpha \beta}(\mathbf{q})$ is a dynamical matrix of ionic plasma oscillations,

$$
D_{i}^{\alpha \beta}(\mathbf{q})=\frac{4 \pi e^{2} Z^{2}}{V_{0}} \sum_{\mathbf{g}} \frac{(\mathbf{q}+\mathbf{g})_{\alpha}(\mathbf{q}+\mathbf{g})_{\beta}}{|\mathbf{q}+\mathbf{g}|^{2}}
$$

Here $V_{0}$ is the volume of the unit cell, $Z$ is a charge of the ion. We use later on self-energy function $\Pi(\mathbf{q} ; \omega)$ of ion vibrations (2) in a harmonic approximation.

The terms $\hat{D}_{i}(\mathbf{q})$ and $\hat{\Pi}(\mathbf{q} ; \omega)$ exactly cancel each other in eq. (2) at $\mathbf{q} \rightarrow 0, \omega \rightarrow 0$. This fact provides a correct description of the acoustic phonons [2, 9].

The generalized dynamic matrix $\hat{D}(\mathbf{q} ; \omega)$ by itself depends on the unknown frequency $\omega$ if you take into account the nonadiabaticity of vibrations. The $\omega-$ dependency comes from the electron contribution

$$
\begin{gathered}
\hat{\Pi}(\mathbf{q}, \omega)=\hat{\Pi}_{1}(\mathbf{q}, \omega)-\hat{\Pi}_{1}(\mathbf{q} \rightarrow 0, \omega \rightarrow 0), \\
\Pi_{1}^{\alpha \beta}(\mathbf{q} ; \omega)=\sum_{\mathbf{g}, \mathbf{g}^{\prime}} V(\mathbf{q}+\mathbf{g}) V\left(-\mathbf{q}-\mathbf{g}^{\prime}\right)(\mathbf{q}+\mathbf{g})_{\alpha}\left(\mathbf{q}+\mathbf{g}^{\prime}\right)_{\beta} \chi\left(\mathbf{q}+\mathbf{g}, \mathbf{q}+\mathbf{g}^{\prime} ; \omega\right),
\end{gathered}
$$

where the $V(\mathbf{q})$ is pseudopotential form-factor, the $\chi\left(\mathbf{q}+\mathbf{g}, \mathbf{q}+\mathbf{g}^{\prime} ; \omega\right)$ is generalized susceptibility of electron gas in a crystal. The susceptibility may be written in terms of electron polarization operator $\pi\left(\mathbf{q}+\mathbf{g}, \mathbf{q}+\mathbf{g}^{\prime} ; \omega\right)$ and inverse dielectric matrix $\epsilon^{-1}\left(\mathbf{q}+\mathbf{g}, \mathbf{q}+\mathbf{g}^{\prime} ; \omega\right)$ in the following way

$$
\chi\left(\mathbf{q}+\mathbf{g}, \mathbf{q}+\mathbf{g}^{\prime} ; \omega\right)=\sum_{\mathbf{g}^{\prime \prime}} \pi\left(\mathbf{q}+\mathbf{g}, \mathbf{q}+\mathbf{g}^{\prime \prime} ; \omega\right) \epsilon^{-1}\left(\mathbf{q}+\mathbf{g}^{\prime \prime}, \mathbf{q}+\mathbf{g}^{\prime} ; \omega\right)
$$

We reduce the analysis of singularities in $\hat{D}(\mathbf{q} ; \omega)$ to the analysis of the ones in the polarization operator $\pi(\mathbf{q}+\mathbf{g}, \mathbf{q}+$ $\left.\mathbf{g}^{\prime} ; \omega\right)$. It will be done below. We have in Hartree approximation for real part of $\pi\left(\mathbf{q}+\mathbf{g}, \mathbf{q}+\mathbf{g}^{\prime} ; \omega\right)$ the following

$$
\pi_{0}\left(\mathbf{q}+\mathbf{g}, \mathbf{q}+\mathrm{g}^{\prime} ; \omega\right)=\frac{2}{N} \sum_{\mathbf{k}, \mathbf{g}^{\prime \prime}} A_{\mathbf{k}}\left(-\mathrm{g}-\mathrm{g}^{\prime \prime}+\mathrm{g}^{\prime}\right) A_{\mathbf{k}+\mathbf{q}+\mathbf{g}+\mathbf{g}^{\prime \prime}}(\mathrm{g}) \frac{n_{\mathbf{k}}-n_{\mathbf{k}+\mathbf{q}+\mathbf{g}+\mathbf{g}^{\prime \prime}}}{E_{\mathbf{k}+\mathbf{q}+\mathbf{g}+\mathbf{g}^{\prime \prime}}-E_{\mathbf{k}}-\hbar \omega}
$$

Here the $A_{\mathbf{k}}(\mathrm{g})$ is a complicated function of the momentum $\mathbf{k}$ (see [10] and below), the $E_{\mathbf{k}}$ is the dispersion low of a band electron. 
The polarization operator singularities are due to the poles of the integrands as follows from eq. (6). The

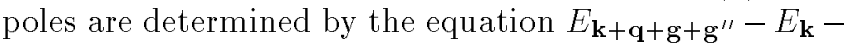
$\hbar \omega=0$. The solutions of this equation give the critical values $k_{c}$ of the integration variable $\mathbf{k}$ at which the $\pi_{0}\left(\mathbf{q}+\mathbf{g}, \mathbf{q}+\mathbf{g}^{\prime} ; \omega\right)$ changes its analytical properties. As a phonon momentum $\mathbf{q}$ is altered, the value of $k_{c}$ changes too. The result is an analytic function of $\mathbf{q}$ if the phonons have momenta $\mathbf{q}$ corresponding to the $k_{c}$ being inside or outside of the integration domain. Singularity appears in the case when the phonons with the $k_{c}$ lies on the boundary of the integration domain $\left(k_{c}=k_{0}\right)$ or falls on the Bragg plane $\left(k_{c}=g / 2\right)$. This fact takes place for the phonon wave vector connecting two points of the Fermi surface with collinear normals [15]. A similar case appears for the Landau singularity in $S$-matrix [16].

As follows from (5) and (6), the nonadiabatisity of states close to the Fermi surface and Bragg plane manifests in two ways. The first effect consists us the appearance of the phonon energy dependence of the dynamic matrix $D^{\alpha \beta}$. The second one is a change of poles of $\pi_{0}\left(\mathbf{q}, \mathbf{q}^{\prime} ; \omega\right)$ (eq. (6)) and the integration domains (over to free electron case). We note that the combinations of $\pi \epsilon^{-1}$ are included in the $\chi\left(\mathbf{q}, \mathbf{q}^{\prime} ; \omega\right)$ and of the $D^{\alpha \beta}(\mathbf{q})$. Therefore, the singularities in $\pi$ are cancelling the singularities in $\epsilon^{-1}$ which have the same form as the ones in $\pi$. It will be demonstrated in paragraph 4 .

We will consider the full operator $\pi$ and its RPA approximation $\pi_{0}$ to have the same singularities. To put it exactly, the $\pi$ and $\pi_{0}$ are different. The full operator $\pi$ contains many-electron contributions. One may investigate the contributions in the local field approximation introducing functions $G(\mathbf{q}, \omega)$ (see e.g. [17]) which depend on the unknown frequency $\omega$ too.

To calculate the diagonal element $\pi_{0}(\mathbf{q}, \mathbf{q} ; \omega)(6)$ a three- wave model is used. The electron waves with the momenta $\mathbf{q}=\mathbf{0}$ and $\mathbf{q}= \pm \mathrm{g} / 2$ only are taken into account in the frames of this model. So,

$$
\begin{aligned}
E_{\mathbf{k}} & =\frac{\hbar^{2}}{2 m}\left(k_{x}^{2}+k_{y}^{2}\right)+E_{\mathbf{g}}^{0}+\operatorname{sign}\left[k_{z}^{2}-(g / 2)^{2}\right] \\
& \times \sqrt{\left[E_{\mathbf{g}}^{0}-\frac{\hbar^{2} k_{z}^{2}}{2 m}\right]^{2}+|v(\mathbf{g})|^{2}} .
\end{aligned}
$$

The coefficients $A_{\mathbf{k}}(\mathrm{g})$ are

$$
\begin{aligned}
& A_{\mathbf{k}}(0)=\frac{\left(E_{\mathbf{k}}-E_{\mathbf{k}-\mathbf{g}}^{0}\right)\left(E_{\mathbf{k}}-E_{\mathbf{k}+\mathbf{g}}^{0}\right)}{\left(E_{\mathbf{k}}-E_{\mathbf{k}-\mathbf{g}}\right)\left(E_{\mathbf{k}}-E_{\mathbf{k}+\mathbf{g}}\right)} \\
& A_{\mathbf{k}}(\mp \mathbf{g})=\frac{v(\mp \mathbf{g})\left(E_{\mathbf{k}}-E_{\mathbf{k} \mp \mathbf{g}}^{0}\right)}{\left(E_{\mathbf{k}}-E_{\mathbf{k} \mp \mathbf{g}}\right)\left(E_{\mathbf{k}}-E_{\mathbf{k} \pm \mathbf{g}}\right)}
\end{aligned}
$$

Here $E_{\mathrm{g}}^{0}=\hbar^{2}(\mathrm{~g} / 2)^{2} / 2 m$, and $v(\mathrm{~g}) \equiv V(\mathrm{~g}) / \tilde{\epsilon}(\mathrm{g})$ is a linearly screened pseudopotential form-factor. Let us use dimensionless variables $z=2 k_{z} / g, x=2 k_{x} / g=$ $\rho \cos \varphi, y=2 k_{y} / g=\rho \sin \varphi$ in a cylinder coordinate system $z, \rho, \varphi$ with the $z$-axis directed along $\mathbf{g}$. We consider the $\mathbf{q}$ to have only two components, $\mathbf{q}=\left(q_{x}, 0, q_{z}\right)$. One guess the equation for the diagonal element after the $\varphi-$ and $\rho$-integrations in eq. (6)

$$
\begin{aligned}
\operatorname{Re} \pi_{0}(\mathbf{Q}, \mathbf{Q} ; \Omega) & =\frac{V_{0} m g}{8 \pi^{2} \hbar^{2} Q_{x}^{2}} \int_{-z_{0}}^{z_{0}} d z\left\{A_{z}(0) A_{z+Q_{z}}(0) f_{1}\left(z, Q_{x}, Q_{z}, \Omega\right)\right. \\
& +A_{z}(0) A_{z-Q_{z}}(0) f_{1}\left(-z, Q_{x}, Q_{z},-\Omega\right)+A_{z}(-2) A_{z+Q_{z}+2}(2) f_{2}\left(z, Q_{x}, Q_{z}, \Omega\right) \\
& +A_{z}(2) A_{z-Q_{z}-2}(-2) f_{2}\left(-z, Q_{x}, Q_{z},-\Omega\right)+A_{z}(2) A_{z+Q_{z}-2}(-2) f_{3}\left(z, Q_{x}, Q_{z}, \Omega\right) \\
& \left.+A_{z}(-2) A_{z-Q_{z}+2}(2) f_{3}\left(-z, Q_{x}, Q_{z},-\Omega\right)\right\} .
\end{aligned}
$$

The functions included in eq. (8) are as follows

$$
\begin{gathered}
f_{1}\left(z, Q_{x}, Q_{z}, \Omega\right)= \begin{cases}B-\operatorname{sign}(B) \sqrt{B^{2}-4 \rho^{2}(z) Q_{x}^{2}}, & B^{2}>4 \rho^{2}(z) Q_{x}^{2}, \\
B, & B^{2} \leq 4 \rho^{2}(z) Q_{x}^{2},\end{cases} \\
B=\operatorname{sign}\left(\left(z+Q_{z}\right)^{2}-1\right) \sqrt{\left(1-\left(z+Q_{z}\right)^{2}\right)^{2}+W_{\mathrm{g}}^{2}}-\operatorname{sign}\left(z^{2}-1\right) \sqrt{\left(1-z^{2}\right)^{2}+W_{\mathrm{g}}^{2}}+Q_{x}^{2}-\Omega, \\
\rho^{2}(z)=\kappa_{F}^{2}-1-\operatorname{sign}\left(z^{2}-1\right) \sqrt{\left(1-z^{2}\right)^{2}+W_{\mathrm{g}}^{2}},
\end{gathered}
$$


where

$$
\begin{aligned}
& \kappa_{F}^{2}=E_{F} / E_{\mathbf{g}}^{0}, \quad W_{\mathbf{g}}=|v(\mathbf{g})| / E_{\mathbf{g}}^{0} \\
& \mathbf{Q}=2 \mathbf{q} /|\mathbf{g}|, \quad \Omega=\hbar \omega / E_{\mathbf{g}}^{0}
\end{aligned}
$$

The function $f_{2}\left(z, Q_{x}, Q_{z}, \Omega\right)$ is obtained from eq. (9) if we replace $z \rightarrow z+2$ in the first term of eq. (10), and the $f_{3}\left(z, Q_{x}, Q_{z}, \Omega\right)$ is obtained by us similar replacement of $z \rightarrow z-2$. The integration limit $z_{0}$ is determined by the condition $\rho^{2}\left(z_{0}\right)=0$.

The integrands in eq. (8) are nonanalytic functions if

$$
B^{2}=4 \rho^{2}(z) Q_{x}^{2}
$$

Eq. (12) is reduced to a well known equation for free electrons $q^{2}-2 k_{F} q=0$ [6] for the spherical Fermi surface.

\section{SINGULARITIES OF SCREANING}

Let us study the behavior of polarization operator in the vicinity of electron topological transition when the Fermi level is located near the face of the Brillouin zone: $\left|1-z_{0}^{2}\right| \sim W_{\mathrm{g}}$. We expand the integrands in eq. (8) on the parameter $\left(1-z^{2}\right) / W_{\mathrm{g}}$. We consider two different cases: $\mathbf{q} \| \mathrm{g}\left(Q_{z} \neq 0, Q_{x}=0\right)$ and $\mathbf{q} \perp \mathrm{g}\left(Q_{z}=0, Q_{x} \neq 0\right)$ calculating the diagonal element $\pi_{0}(\mathbf{Q}, \mathbf{Q} ; \Omega)$.

A. In the case of $\mathbf{q} \| \mathbf{g}$ and $q_{z}=g-\delta q_{z}, \delta q_{z} \ll g\left(Q_{z}=\right.$ $\left.2-\delta Q_{z}\right)$ the presence of nonadiabatic states in the vicinity of electron topological transition leads to anomalous logarithm contribution in the $\pi_{0}(\mathbf{Q}, \mathbf{Q} ; \Omega)$ [14]. This contribution is absent in adiabatic theory. Its magnitude depends on the Fermi surface topology and its maximum value is

$$
\delta \pi_{0}(Q, Q ; \Omega) \simeq \frac{V_{0} \mathrm{mgW} \mathrm{W}_{\mathrm{g}} s}{64 \pi^{2} \hbar^{2}} \ln \left|\frac{\delta Q_{z}-\delta Q_{c}}{\delta Q_{z}+\delta Q_{c}}\right|
$$

when electron pocket in the second Brillouin zone appears (in eq. (13) $s=\Omega / \delta Q_{z}$ is phase velocity of phonons and $\left.\delta Q_{c}=W_{\mathrm{g}} s / 2\right)$. At small momenta $Q_{z}=\delta Q_{z}$ umklapp processes give the same contribution with the opposite sign. So, the terms $\delta \pi_{0}$ (eq. (13)) is are absent in the $\pi_{0}(\mathbf{Q}, \mathbf{Q} ; \Omega)$. Here is only an ordinary $\left(1-q_{z} / 2 k_{F}\right) \ln \mid$ $1-q_{z} / 2 k_{F} \mid$ Kohn singularity. It exists at any position of $E_{F}$ relatively to a Brillouin zone face. Unlike of the Kohn singularity, logarithm one at $\delta Q_{z} \rightarrow \delta Q_{c}\left(\delta \pi_{0}\right.$, eq. (13)) does not depend directly on the $k_{F}$ and it is due to nonadiabatic states of the electron-ion system. We note that the difference of the coefficients $A_{\mathbf{k}}(0)$ from 1 and $A_{\mathbf{k}}( \pm \mathrm{g})$ from 0 is essential in the calculation of the $\pi_{0}(\mathrm{Q}, \mathrm{Q} ; \Omega)$ because it is the difference that leads to the cancellation of the logarithm contribution at small momenta $Q_{z}$.
Nondiagonal elements $\pi_{0}\left(\mathbf{q}+\mathbf{g}, \mathbf{q}+\mathbf{g}^{\prime} ; \omega\right)$ also contain the terms of the form of eq. (13) having the same order of magnitude as the diagonal ones. The difference from the case of $\pi_{0}(\mathbf{Q}, \mathbf{Q} ; \Omega)$. The nondiagonal parts of the polarization operator contain the logarithm singularity both in the region of momenta $q_{z} \sim g$ and in that of $q_{z} \ll g$.

Phonon energy spectrum has no logarithm singularity due to pseudopotential screening. The $\pi(\mathbf{q} ; \omega)$ and $\epsilon(\mathbf{q} ; \omega)$ contain the logarithm singularity $\delta \pi_{0}($ eq. $(13))$ since the $\chi(\mathbf{q} ; \omega) \sim \pi(\mathbf{q} ; \omega) \epsilon^{-1}(\mathbf{q} ; \omega)(\epsilon(\mathbf{q} ; \omega)=1+$ $\left.v_{c}(\mathbf{q}) \pi(\mathbf{q} ; \omega) ; v_{c}(\mathbf{q} ; \omega)=4 \pi e^{2} / \mathbf{q}^{2}\right)$. These divergences are cancelling each other at $\delta q_{z} \rightarrow \delta q_{c}$. Pole anomaly only is present in group velocity of phonons like it does in the case of near free electrons [9].

B. In the case of $\mathbf{q} \perp \mathrm{g}$ a nonanalyticity is observed in polarization operator as a function of $q_{x}$ in the vicinity of electron topological transition. The main contribution to the integral of eq. (8) comes from its first pair of terms corresponding to the normal processes. It is due to the $A_{z}^{2}(0) \sim 1$ in the whole integration on the $z$ domain. We have the $A_{z}( \pm 2) A_{z \pm 2}(\mp 2) \sim 1$ only in the small vicinity $\sim W_{\mathrm{g}}$ near the Brillouin zone face $z= \pm 1$ for the second and the third pairs of terms which are due to umklapp processes . The $A_{z}( \pm 2) A_{z \pm 2}(\mp 2) \sim W_{\mathrm{g}}^{2}$ in major part of the integration domain.

For the closed Fermi surface the values of $\pi_{0}(\mathbf{q}, \mathbf{q} ; \omega)$ at $q_{x} \rightarrow s-0$ and at $q_{x} \rightarrow s+0$ are different. Their difference is

$$
\begin{aligned}
& -\left(4 Q_{x}\right)^{-1} \sqrt{W_{\mathrm{g}} / 2}\left[\left(Q_{x}-s\right)^{2}+d_{0}^{2}\right] \\
& \times \arccos \frac{d_{0}}{\left(\left(Q_{x}-s\right)^{2}-d_{0}\right)^{1 / 2}}
\end{aligned}
$$

In eq. (14) $d_{0}=2 \sqrt{\kappa^{(1)}-\kappa_{F}^{2}}, s=\Omega / Q_{x}$ is phonon phase velocity, $\kappa^{(1)}=1-W_{\mathrm{g}}$ is the energy at which the Fermi surface is changed from closed to open. The magnitude of the jump in the polarization operator is determined by eq. (14). The singular point $Q_{x}=s$ is essentially determined by nonadiabatic states, and there is no such singularity in adiabatic approximation. The case of $Q_{x}=0$ when polarization operator has logarithm contribution is exclusive, as mentioned above.

For the open Fermi surface two different cases are possible depending on the ratio of phonon phase velocity, $s=\Omega / Q_{x}$, and the diameter of the Fermi surface "neck", $d_{1}=\sqrt{\kappa_{F}^{2}-\kappa^{(1)}}$. The nonanalyticity is brought to the $\pi_{0}(\mathbf{Q}, \mathbf{Q} ; \Omega)$ both by normal processes and umklapp ones if $s<d_{1}$. However, umklapp processes contribute only before the appearance of the second Brillouin zone in a narrow strip of the parameter $d_{1}$ values, $\sqrt{8 W_{\mathrm{g}}}-s<d_{1}<\sqrt{8 W_{\mathrm{g}}}+s$. For normal processes there are two values of phonon momentum, $Q_{x}=d \pm s$, at which the critical points $z_{c}$ fall on the boundary of the integration domain $\left(z_{0}=1\right)$. Polarization operator of eq. (8) is of the form 


$$
\pi_{0}(\mathbf{Q}, \mathbf{Q} ; \Omega) \simeq \frac{V_{0} m g}{8 \pi^{2} \hbar^{2}} \begin{cases}1, & Q_{x} \leq d_{1}-s \\ 1-\alpha F_{1}, & d_{1}-s<Q_{x} \leq d_{1}+s \\ 1-\alpha\left(F_{1}-2 s\right), & Q_{x}>d_{1}+s\end{cases}
$$

Here $\alpha=\frac{\pi}{8} \sqrt{W_{\mathrm{g}} / 2}$ and $F_{1}=\left[\left(Q_{x}+s\right)^{2}-d_{1}^{2}\right] Q_{x}^{-1}$.

Such a behavior of $\pi_{0}(\mathbf{Q}, \mathbf{Q} ; \Omega)$ means that the jumps with the magnitude $\sim-\pi \sqrt{W_{\mathrm{g}} / 2}$ are contained in the derivative $\partial \pi_{0}(\mathbf{Q}, \mathbf{Q} ; \Omega) / \partial \Omega$ at the momenta $Q_{x}=d_{1} \mp \mathbf{s}$. Umklapp processes give nonanalytic contribution of the magnitude $\sim a^{2} \ln a$ to the $\pi_{0}(\mathbf{Q}, \mathbf{Q} ; \Omega)$ but respective jump in the derivative is $\sim a \ll \sqrt{2 W_{\mathrm{g}}}$. The limit of adiabatic approximation is possible in this case. The single value of momentum $Q_{x}=d_{1}$ for which the $z_{c}$ falls on the boundary of integration domain exists only. The curve of the $\pi_{0}(\mathbf{Q}, \mathbf{Q} ; \Omega)$ versus $Q_{x}$ has a break at this point. The condition of $s<d_{1}$ may be performed, practically, for any Fourier transform $W_{\mathrm{g}}$ of a pseudopotential since at small phonon momenta the value of $s$ is two or three orders less than $\sqrt{W_{\mathbf{g}}}$.

The case of $s>d_{1}$ may be realised at the moment of forming an open Fermi surface. Then

$$
\pi_{0}(\mathbf{Q}, \mathbf{Q} ; \Omega) \simeq \frac{V_{0} m g}{8 \pi^{2} \hbar^{2}} \begin{cases}1-4 \alpha s, & Q_{x}<s-d_{1}, \\ 1-\alpha F_{1}, & s-d_{1} \leq Q_{x} \leq s+d_{1} \\ 1-2 \alpha\left(F_{1}-2 s\right), & Q_{x}>s+d_{1} .\end{cases}
$$

The kind of behavior of the $\pi_{0}(\mathbf{Q}, \mathbf{Q} ; \Omega)$ is changed to some extent. However, the magnitude of the jump in the $\partial \pi_{0}(\mathbf{Q}, \mathbf{Q} ; \Omega) / \partial \Omega$ has the same order as that for normal processes at $s<d_{1}$. The case of $s>d_{1}$ is essentially nonadiabatic and here is not a limit of adiabatic approximation. The condition of $s>d_{1}$ constrains strongly the area of the Fermi energy values: $4\left(\kappa_{F}^{2}-\kappa^{(1)}\right)<\Omega$.

An additional parameter, $d_{2}=2 \sqrt{\kappa_{F}^{2}-\kappa^{(2)}}\left(d_{2}\right.$ is the diameter of the cavity) appears in theory along with $s$ and $d_{1}$ with the formation of the electron cavity in the second Brillouin zone. The $\kappa^{(2)}=1+W_{\mathbf{g}}$ is the energy of appearing electron topological transition related to filling the second Brillouin zone. The six different cases are possible depending on the ratio between the parameters $d_{1}$ and $d_{2}$. The cases of great interest are those which admit of being realized independently from the magnitude of $W_{\mathbf{g}}$. Thus, for $s<d_{2}, 2 s<\left(d_{1}-d_{2}\right)$ there are four critical values of phonon momentum, $Q_{x}=d_{2} \mp s$ and $Q_{x}^{\prime}=d_{2} \mp s$. For them critical values $z_{c}$ of integration variable $z$ get on the Brillouin zone face, and analytical properties of the $\pi_{0}(\mathbf{Q}, \mathbf{Q} ; \Omega)$ are changed

$$
\pi_{0}(\mathbf{Q}, \mathbf{Q} ; \Omega) \simeq \frac{V_{0} m g}{8 \pi^{2} \hbar^{2}}\left\{\begin{array}{l}
L_{1}, Q_{x}<d_{2}-s \\
L_{2}, d_{2}-s \leq Q_{x}<d_{2}+s \\
L_{3}, d_{2}+s \leq Q_{x} \leq d_{1}-s \\
L_{4}, d_{1}-s<Q_{x} \leq d_{1}+s \\
L_{5}, Q_{x}>d_{1}+s
\end{array}\right.
$$

where

$$
\begin{aligned}
& L_{1}=1+2 \alpha\left(d_{2}+F_{2}+F_{3}\right) \\
& L_{2}=1+\alpha\left(d_{2}\left(3+\frac{s}{Q_{x}}\right)+2 F_{2}\right) \\
& L_{3}=1+4 \alpha d_{2} \\
& L_{4}=1+\alpha\left(d_{2}\left(3-\frac{s}{Q_{x}}\right)-\pi F_{1}+2 F_{2}\right), \\
& L_{5}=1+2 \alpha\left(d_{2}+F_{2}+F_{3}-\pi\left(F_{1}-2 s\right)\right) \\
& F_{2}=\left(4 Q_{x}\right)^{-1}\left[\left(Q_{x}-s\right)^{2}-d_{2}^{2}\right] \ln \left|\frac{d_{2}-Q_{x}+s}{d_{2}+Q_{x}-s}\right| \\
& F_{3}=\left(4 Q_{x}\right)^{-1}\left[\left(Q_{x}+s\right)^{2}-d_{2}^{2}\right] \ln \left|\frac{d_{2}-Q_{x}-s}{d_{2}+Q_{x}+s}\right| .
\end{aligned}
$$

The jump of the derivatives $\partial \pi_{0}(\mathbf{Q}, \mathbf{Q} ; \Omega) / \partial Q_{x}$ is $\sim W_{\mathbf{g}}^{1 / 2}$. Umklapp processes give small $\left(\sim a^{2} \ln a\right)$ contribution 


\section{E. V. ZAROCHENTSEV}

like they do in the case of the open Fermi surface. The adiabatic limit is possible at $s \rightarrow 0$. The violation of the analytic behavior of polarization operator appears at two values of the momentum $Q_{x}=d_{2}$ and $Q=d_{1}$ only.

As the second Brillouin zone is being filled the case of $d_{2}<s<d_{1}, 2 s<\left(d_{1}-d_{2}\right)$ may be performed into reality. Polarization operator is of the form

$$
\pi_{0}(\mathbf{Q}, \mathrm{Q} ; \Omega) \simeq \frac{V_{0} m g}{8 \pi^{2} \hbar^{2}} \begin{cases}L_{3}, & Q_{x} \leq s-d_{2}, s+d_{2} \leq Q_{x} \leq d_{1}-s \\ L_{2}, & s-d_{2}<Q_{x}<s+d_{2} \\ L_{4}, & d_{1}-s<Q_{x} \leq d_{1}+s \\ L_{5}, & Q_{x}>d_{1}+s\end{cases}
$$

In this case the moment of violation of the condition $d_{2}<s$ appears as the second Brillouin zone is being filled. Thus, the last case is possible only at early stage of forming electron cavity, i.e. in the narrow region of the Fermi energy values when $4\left(\kappa_{F}^{2}-\kappa^{(2)}\right)<\Omega$. Other four cases may occur for quite a weak $W_{\mathrm{g}}: v(\mathrm{~g}) / E_{F} \sim$ $\left(10^{-4}-10^{-6}\right)$.

Nondiagonal elements $\pi_{0}\left(\mathbf{q}+\mathbf{g}, \mathbf{q}+\mathbf{g}^{\prime} ; \omega\right)$ of the polarization operator appear to have an order of magnitude $v(\mathrm{~g})$ but they contain similar singularities to chose of $\pi_{0}(\mathbf{q}, \mathbf{q} ; \omega)$.

Numerical calculation shows all the things said above to be valid also in the case of am arbitrary direction of $\mathbf{q}$. With increasing $q_{z}$ the angle between right and left tangents at the point of break decreases. The values of $q_{x}$ and $q_{z}$ are correspondent to nondiametral Kohn singularities [7] at the break points on the curves of $\pi_{0}(\mathbf{q}, \mathbf{q} ; \omega)$. The presence of breaks on the curves of $\pi_{0}(\mathbf{q}, \mathbf{q} ; \omega)$, and, therefore, on the dispersion curves too, gives rise to the jumps in momentum derivatives of $\pi_{0}(\mathbf{q}, \mathbf{q} ; \omega)$.

Similar jumps must be observed also in the phonon group velocity [4].

\section{PHONON GROUP VELOCITY ANOMALIES}

The Green function poles determine a nonlinear equation

$$
\omega^{2} \delta_{\alpha \beta}-D_{i}^{\alpha \beta}(\mathbf{q})-\operatorname{Re} \Pi^{\alpha \beta}(\mathbf{q}, \omega)=0
$$

for the phonon frequency $\omega$ (their dumping is not considered here). The equation for the polarization operator can be found everywhere (see, e.g., [9]), and we don't write it. The singularities on the phonon dispersion curves considered here have the same genesis as the Kohn ones [4] but the presence of the Bragg plane leads to drastic changes in their behavior and location.

Let us to be interested in the region of $q \sim 2 k_{F}$. We consider that $\operatorname{Re} \Pi(\mathbf{q}, \omega)$ neglecting umklapp processes $(\mathrm{g}=0)$ escape formulas complication. As it follows from the equation for $\Pi^{\alpha \beta}(\mathbf{q}, \omega)$ (eqs. (3), (4)), the terms with $\mathrm{g} \neq 0$ contain the values of pseudopotential form-factor with the arguments $\mathbf{q}+\mathbf{g}$. The values drop rapidly with the growing of its arguments. In the case of $\mathrm{g}=0$ the phonon dispersion low can be easily obtained from (19) in the form

$$
\Omega^{2}=\Omega_{p l}^{2}+B \alpha x^{2} \frac{\pi_{0}(x, \Omega)}{\epsilon_{0}(x, \Omega)}
$$

The dimensionless variables were used in (20)

$$
\begin{aligned}
& \Omega=\frac{\omega m}{2 \hbar k_{F}^{2}} ; \quad x=\frac{q}{2 k_{F}} ; \\
& \Omega_{p l}=\frac{\omega_{p l} m}{2 \hbar k_{F}^{2}} ; \\
& \epsilon_{0}(x, \Omega)=1+v_{c}(x) \pi_{0}(x, \Omega) ; \\
& B=|V(\mathbf{x})|^{2}\left(\frac{m}{2 \hbar^{2} k_{F}^{2}}\right)^{2},
\end{aligned}
$$

where $\omega_{p l}$ is the ion plasma frequency, $v_{c}(x)$ is Coulomb form-factor, $V(\mathbf{x})$ is the pseudopotential. In eq. (20) the magnitude of $\alpha$ is proportional to the electron-phonon interaction vertex and its form is not important here.

It is enough for our purpose to use the Lindhard form for polarization operator $\pi_{0}(x, \Omega)$

$$
\begin{aligned}
\pi_{0}(x, \Omega) & =1-\frac{1}{4 x} \frac{\Omega^{2}+x^{4}-x^{2}}{x^{2}} \\
& \times \ln \left|\frac{[\Omega-x(x+1)][\Omega+x(x+1)]}{[\Omega-x(x-1)][\Omega+x(x-1)]}\right| \\
& -2 \Omega \ln \left|\frac{[\Omega-x(x+1)][\Omega+x(x-1)]}{[\Omega-x(x-1)][\Omega+x(x+1)]}\right| .
\end{aligned}
$$

The dependence of $\pi_{0}$ on $\Omega$ is neglected in the adiabatic approximation. Thus, the $\Omega(x)$ has only Kohn singularity $x \ln x$, which leads to logarithm divergence of the phonon group velocity at $x \rightarrow \pm 1\left(q \rightarrow \pm 2 k_{F}\right)$. With considering electron nonadiabatic states, one may not divide the energy of the system on electron and ion parts. Elementary excitations spectrum should be found by solving a common system of equations. It leads to a nonlinear behavior of $\Omega(x)(20)$, with the phonon group velocity $w$ 


$$
\frac{\partial \Omega}{\partial x}=\frac{A_{1}+A_{2}}{A_{3}}
$$

being the function not only of the $\partial \pi_{0} / \partial x$ (like it does in the adiabatic approximation) but of the $\partial \pi_{0} / \partial \Omega$. In equation (23)

$$
\begin{aligned}
A_{1} & =\frac{\alpha B}{2 \epsilon_{0}^{2}(x, \Omega)} \frac{\Omega^{2}-x^{2}}{x^{2}} \ln \left|\frac{[\Omega-x(x+1)][\Omega+x(x+1)]}{[\Omega-x(x-1)][\Omega+x(x-1)]}\right|, \\
A_{2} & =\frac{\partial \Omega_{p l}^{2}}{\partial x} \frac{\alpha \pi_{0}(x, \Omega)}{\epsilon_{0}(x, \Omega)} \frac{\partial B}{\partial x} x^{2}+\frac{2 \alpha B x}{\epsilon_{0}^{2}(x, \Omega)} \frac{2 \alpha \pi_{0}(x, \Omega) B x}{\epsilon_{0}^{2}(x, \Omega)} \\
& -\frac{\alpha B \pi_{0}(x, \Omega) x}{\epsilon_{0}^{2}(x, \Omega)}+\frac{2 \alpha B v_{c}(x) \pi_{0}^{2}(x, \Omega) x}{\epsilon_{0}^{2}(x, \Omega)}, \\
A_{3} & =2 \Omega+\frac{\alpha B}{\epsilon_{0}^{2}(x, \Omega)} \frac{\Omega}{x} \ln \left|\frac{[\Omega-x(x+1)][\Omega+x(x+1)]}{[\Omega-x(x-1)][\Omega+x(x-1)]}\right| \\
& -\frac{\alpha B}{\epsilon_{0}^{2}(x, \Omega)} x \ln \left|\frac{[\Omega-x(x+1)][\Omega+x(x-1)]}{[\Omega-x(x-1)][\Omega+x(x+1)]}\right| .
\end{aligned}
$$

The Kohn anomaly is canceled in group velocity since the $A_{1}$ term (containing logarithm Kohn singularity in adiabatic approximation) and the $A_{3}$ one have the same singularities. One can easily see it by transforming of the term of $A_{1} / A_{3}$ near $x=1$ to the form

$$
\frac{A_{1}}{A_{3}}=\frac{x^{2}-\Omega^{2}}{2 \Omega}\left[-1+\frac{C-x \ln \left|\frac{\Omega+x-1}{\Omega-x+1}\right|}{C-\Omega \ln |[\Omega-x+1][\Omega+x-1]|-x \ln \left|\frac{\Omega+x-1}{\Omega-x+1}\right|}\right],
$$

where $C=2 \Omega \epsilon_{0}^{2}(x, \Omega) /(\alpha B)$.

The numerator and denominator in eq. (27) contain the same logarithm singularities. The group velocity has finite limits which equal $\pm v_{F}$ in the points of $x=1 \pm \Omega$ where the logarithms diverge ( $v_{F}$ is the electron velocity on the Fermi surface)

$$
\lim _{x \rightarrow 1-\Omega} \frac{\partial \omega(\mathbf{q})}{\partial q}=-v_{F} ; \quad \lim _{x \rightarrow 1+\Omega} \frac{\partial \omega(\mathbf{q})}{\partial q}=v_{F}
$$

The singularities due to denominator $A_{3}$ tending to zero appear in the phonon group velocity instead logarithm ones. It is essential that the zero values of $A_{3}$ are located between the zero values of the second term in (27). This fact determines the behavior of the function $A_{1} / A_{3}$

In eq. (23) the second term $A_{2} / A_{3}$ also has singularities due to zero values of the denominator $A_{3}$. The behavior of this term depends on the sign of $A_{2}(25)$ which is the function of the $\partial \omega_{p l} / \partial q$ and $\partial V(\mathbf{q}) / \partial \mathrm{q}$.

Thus, the phonon group velocity can be represented as a sum of two competing terms (see eq. (23)) which have the same singularities related to the tending of the denominator to zero. We note that the denominator in group velocity is equal to twin adiabatic frequency and has no singularities in adiabatic approximation.

As is seen from eqs. (24), (26), the singularities of phonon group velocity are shifted to large momenta relatively to $q=2 k_{F}$ on the value of $\Delta q=\omega m /\left(\hbar k_{F}\right)$. The distance between zero points of the denominator in eq. (23) $\left(x_{2,3} \approx 1+\Omega \pm 2 \Omega \exp (-C)\right)$ depends on the magnitude of $C$ (eq. (27)) significantly. The $C \geq 10^{2}$, $B \leq 10^{-2}$ for simple metals since $B \sim\left|V\left(q=2 \bar{k}_{F}\right)\right|^{2}$. There is a hope that the factor $\exp (-C)$ has a larger magnitude due to the presence of $d$ - or $f$-electrons in the transition metals and the rare-earth ones.

\section{CONCLUSION}

The dynamical problem is investigated in this paper, with the dynamical matrix $\mathbf{D}(\mathbf{q}, \omega)$ being calculated in the three-wave model for a crystal when the Fermi surface is close to the Bragg plane $\left|E_{F}-E_{\mathbf{g}}^{0}\right| \leq \hbar \omega_{D}$. Major attention is given to the analysis of the polarization operator $\pi\left(\mathbf{q}, \mathbf{q}^{\prime} ; \omega\right)$ because the lattice vibrational spectrum singularities of a metal or narrow-gap semiconductor are due to singularities in screening. The electron topological transitions are the phenomena for which the consideration of nonadiabatic states (the influence of electronphonon interaction on them) is essential when the type 


\section{E. V. ZAROCHENTSEV}

of anomalies and singularities is being found.

The nonlinearity of equations for lattice vibration frequencies is caused by retarded behavior of electronion potential at vibrations. The qualitative changing of group velocity behavior takes place in the vicinity of Kohn singularity momenta $q \sim 2 k_{F}$ although the magnitude of phonon frequencies is changed insignificantly over the adiabatic ones. It should be emphasized that the group velocity anomalies in this case are already not Kohn ones both of the type of singularity and of its genesis. They are due not only to harsh edge of Fermi-Dirac distribution but to the change of the distribution near the Bragg plane. Phonon frequency dependence of the polarization operator must lead to new effects in thermodynamical and kinetic properties of metals and semiconductors. Unfortunately, we don't know any such experiments to be carried out at the present time.

The polarization operator singularities appear if the critical values of the integrand (the points of changing its analytical behavior) fall on the bound of the integration domain or on the Bragg plane.

For closed Fermi surface $\left(\kappa_{F}^{2}<\kappa^{(1)}\right)$ there is one value of $Q_{x}=s$, at which the condition $z_{c}=z_{0}$ is fulfilled. It is the point in which the changing of the analytical behavior of $\pi_{0}(\mathbf{q}, \mathbf{q} ; \omega)$ takes place. The existence of a singular point $Q_{x}=s$ is related to the presence of nonadiabatic electron states in the reconstructed area, i.e. in the layer with the width $\sim \hbar \omega$ near the Fermi surface. The electron-phonon interaction leads in this layer to the transitions in the electron spectrum with the phonon emission or absorption. The $\omega=0, s=0$ in the adiabatic approximation, and the integral (8) has no singularities at $Q_{x} \neq 0, Q_{z}=0$.

The processes with phonon emission and absorption appear to be nonequivalent ones because the condition of $z_{c}=z_{0}$ is fulfilled only for the process with the phonon absorption. Thus, it is the absorption process that abides by the existence of the polarization operator singularity.

Two cases are possible depending on the ratio between the phonon phase velocity $s$ and the neck diameter $d_{1}$ when the electron neck is formed for the open Fermi surface $\left(1-\kappa^{(1)}\right) \leq \kappa_{F}^{2} \leq \kappa^{(2)}$. In the case of $s<d_{1}$ there are two values of momentum $d_{1} \pm s$ at which the analytical behavior of the $\pi_{0}(\mathbf{q}, \mathbf{q} ; \omega)$ is changed. The limit of adiabatic approximation is possible here. The case of $s>d_{1}$ is nonadiabatic essentially.

When the electron cavity exists in the second Brillouin zone $\left(\kappa_{F}^{2}>\kappa^{(2)}\right)$, the singularities in the $\pi_{0}(\mathbf{q}, \mathbf{q} ; \omega)$ appear if the critical values fall on the Fermi surface $\left(z_{c}=z_{0}\right)$ or on the Bragg plane $\left(z_{c}=1\right)$. Ten different cases of behavior of integral (8) are possible depending on the ratio among the $s, d_{1}$ and $d_{2}$. The adiabatic limit is possible in the case of $s<d_{2}<d_{1}, 2 s<\left(d_{1}-d_{2}\right), 2 s<d_{2}$ only. Here is the degeneration of critical points to one point. The change of analytical behavior of the integral takes place for two values of the momentum $Q_{x}=d_{1}$ and $Q_{x}=d_{2}$ only. At these values the momentum $\mathbf{Q}$ connects the states on the Bragg plane which belong to the reconstructed area of electron spectrum.

The Fermi surface topology changing similar to the one considered here takes place in In [18] and Al [19] under pressure. One may expect the appearing of the singularities in the phonon group velocity in these metals, and also in thermo-E. M. F. [20].

The author is grateful to Dr. I. E. Dragunov for collaboration and also to Yu. V. Eremeichenkova for help in preparing the manuscript and for useful comments.
[1] E. G. Maksimov, D. Yu. Savracov, S. Yu. Savrasov, Usp. Fiz. Nauk 167, 353 (1996).

[2] E. G. Brovman, Yu. M. Kagan, Usp. Fiz. Nauk 112, 369 (1974).

[3] B. T. Geilikman, Usp. Fiz. Nauk 115, 403 (1975).

[4] W. Kohn, Phys. Rev. Lett. 2, 393 (1959).

[5] B. N. Brockhouse, K. R. Rao, A. D. B. Woods, Phys. Rev. Lett. 7, 93 (1961).

[6] E. J. Woll, W. Kohn, Phys. Rev. 126, 1693 (1962).

[7] Yu. M. Kagan, V. V. Pushkarev, A. Kholas, Zh. Eksp. Teor. Fiz. 84, 1494 (1983).

[8] J. W. Weymouth, R. Stedman, Phys. Rev. B 2, 4743 (1970).

[9] I. E. Dragunuv, E. V. Zarochentsev, S. M. Orel, Fiz. Met. Metalloved. 87, 837 (1989).

[10] I. E. Dragunov, Ph.D. thesis, Donetsk (1992).

[11] V. V. Nemoshkalenko, A. V. Zhalko-Titarenko, A. Ya. Perlov, et al., Metallofizika 13, 114 (1991).
[12] Y. Nakagowa $Y$, A. D. B. Woods, Phys. Rev. Lett. 11, 271 (1963).

[13] B. N. Powell, P. Martel, A. D. B. Woods, Phys. Rev. 171, 727 (1968).

[14] I. E. Dragunov, E. V. Zarochentsev, S. M. Orel, Fiz. Tverd. Tela 31, 314 (1990).

[15] P. L. Taylor, Phys. Rev. 131, 1995 (1963).

[16] V. B. Berestetskiy, E. M. Lifshits, L. P. Pitaevskiy, Kvantovaia eliektrodinamika (Quantum electrodynamics) (Nauka, Moskow, 1980).

[17] M. V. Vavrukh, Fiz. Nizk. Temp. 22, 1005 (1996).

[18] I. Ya. Volynskiy, V. I. Makarov, V. V. Gann, Zh. Eksp. Teor. Fiz. 69, 1019 (1975).

[19] D. R. Overcash, Tacy Davis, J. W. Jr. Cook, M. J. Skove, Phys. Rev. Lett. 46, 287 (1981).

[20] N. V. Zavoritskiy et al., Zh. Eksp. Teor. Fiz. 94, 344 (1988). 


\title{
ВПЛ ИВ НЕАДІЯБАТИЧНИХ СТАНІВ, БЛИЗЬКИХ ДО ГРАНІ ЗОНИ БРИЛЛЮЕНА, НА КОЛ ИВНИЙ СПЕКТР
}

\author{
Є. В. Зароченцев \\ Донецьвкий фізико-технічний інститут Національной Академій Наук Украйни \\ вул. Р. Люксембур , 72, Донеиьк, 340114, Украйна \\ E-mail: zero@host.dipt.donetsk.ua
}

Ва жливу роль у формулюванні особливостей коливного спектра кристала відіграють дві обставини: неадіябатичні стани електрон-йонної системи, що ле жать поблизу граничної енертї̈, і близькість цих станів до Бреггової площини. В околі електронного топологічного переходу в упорядкованих середовищах наявність неадіябатичних станів приводить до радикальної зміни характеру недіяметральних особливостей. Найсильніше цей ефект виявляється тоді, коли імпульс фонона з'єднує дві перебудовані поблизу Брегтової площини області електронного спектра. Установлено умови, при яких деякі особливості поляризаційного оператора приводять до неаналітичної зале жности групової швидкости фонона від імпульсу. 